\title{
A Two-Column Flash Chromatography Approach to Pyoverdin Production from Pseudomonas putida GB1
}

by

Owen W. Duckworth ${ }^{*, 1}$, Dawn S. Markarian ${ }^{1}$, Dorothy L. Parker ${ }^{2}$, and James M. Harrington ${ }^{3}$

${ }^{1}$ Department of Crop and Soil Sciences, North Carolina State University, Raleigh, NC 27695, USA

${ }^{2}$ Scripps Institution of Oceanography, University of California, San Diego, La Jolla, California 92093, USA

${ }^{3}$ RTI International, 3040 East Cornwallis Road, Durham, NC 27709, USA

Submitted as a short communication to

Journal of Microbial Methods

January 27, 2017

Submitted in Revised form

January 30, 2017

*To Whom Correspondence Should be Addressed

owen_duckworth@ncsu.edu

Tel. (919) 513-1577

Fax. (919) 515-1267 


\section{Graphical Abstract:}

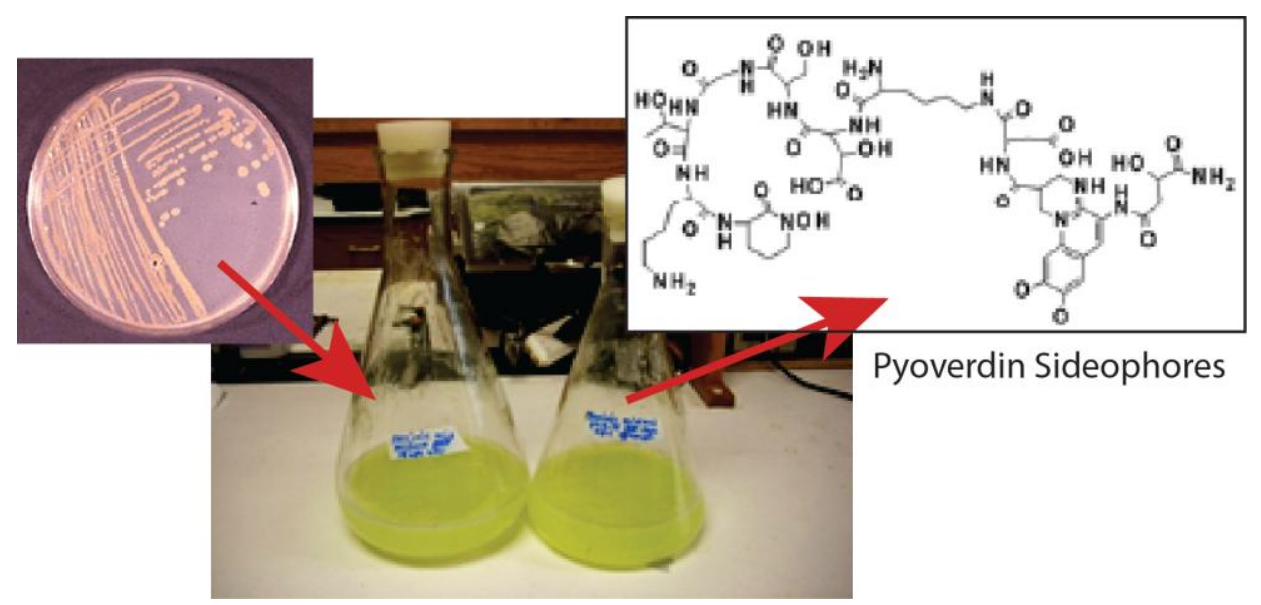

Keywords: Siderophores, Purification, Flash chromatography

Abstract. Our knowledge of the biological and environmental reactivity of siderophores is limited by the difficulty and cost of obtaining reasonable quantities by purification or synthesis. In this note, we describe a modified procedure for the low-cost, mg-scale purification of pyoverdin-type siderophores using a dual-flash chromatography (reverse-phase absorption and size exclusion) approach. 
Text. Siderophores are a diverse class of chelating agents produced by bacteria, fungi, and grasses to promote uptake of iron and other bioinaccessible nutrient metals (Kraemer et al., 2014; Kraepiel et al., 2009; Neilands, 1995). The pyoverdins (PVDs), a group of fluorescent molecules that are produced by certain bacteria in the Pseudomonas genus and characterized by a fluorescent quinoline chromophore (Budzikiewicz, 2010), have been associated with biological and environmental phenomena beyond metal uptake, including pathogenesis, plant disease suppression, contaminant transport, and biomineral formation (Edberg et al., 2010; Lemanceau et al., 2009; Meyer, 2000; Parker et al., 2007).

Currently, PVDs are only available in small quantities at high costs, and thus larger quantities are required for many biological and environmental studies must be produced by purification, e.g., via size exclusion chromatography (Meyer and Abdallah, 1978). In this communication, we describe the application of flash chromatography (compressed gas driven) to allow for PVD production with low-cost chromatographic separation (Still et al., 1978). In addition, we improve upon this methodology by incorporating a desalting/concentrating reverse phase column (Parker et al., 2004).

Pseudomonas putida GB1 cultures (Parker et al., 2004) were grown from frozen stock on half-strength Luria broth (LB) agar plates. A $50 \mathrm{ml}$ iron-free succinate defined medium (SDM) (Meyer et al., 1997) "starter" was inoculated with colonies from the plate and allowed to grow for $24 \mathrm{hr}$ at $28^{\circ} \mathrm{C}$. A $10 \mathrm{ml}$ starter aliquot was then used to inoculate $1 \mathrm{~L}$ of SDM in sterile Erlenmeyer flasks. The flasks were shaken for $96 \mathrm{hrs}$ at $120 \mathrm{rpm}$ (in stationary phase), and then visually assessed (both by a yellow-green color in the visible and by fluorescence when exposed to a UV-A light) to qualitatively verify PVD production. The culture was distributed into HDPE bottles and centrifuged at $3825 \mathrm{~g}$ RCF 
for 20 min. The supernatant was decanted and vacuum filtered (Whatman 42) to remove solids to prevent column clogging.

The filtered medium was passed through a flash solid phase extraction (SPE) column (Amberlite XAD-4) to desalt and concentrate the sample. To facilitate sorption to the stationary phase, resin and the filtrate were evenly distributed into foil-coated HDPE bottles, which were shaken at $220 \mathrm{rpm}$ for $24 \mathrm{hr}$. The solids and media were transferred to a $5 \times 30 \mathrm{~cm}$ column, drained by gravity, washed by flash chromatography with $1.5 \mathrm{~L}$ of deionized water under 3.4 atm $\mathrm{N}_{2}$, and then the eluent was changed to a 50/50\% vol mixture of methanol and water. In all cases where glassware is pressurized, additional shielding (a hood or column guard) was employed. The first $150 \mathrm{ml}$ of eluent was discarded, and the following $600-750 \mathrm{ml}$ of eluent was collected (until eluent ran clear). The resin was regenerated by rinsing with $1.5 \mathrm{~L}$ methanol. The collected eluent was concentrated by rotary evaporation at $60^{\circ} \mathrm{C}$ (IKA V R10 basic Rotovap), resulting in a final extract volume of $10-15 \mathrm{ml}$.

The concentrate was further purified by size exclusion flash chromatography. A $2.5 \times 40 \mathrm{~cm}$ column (CM-sephadex G-25, General Electric) was preconditioned by flushing with $400 \mathrm{ml}$ of $50 \mathrm{mM}$ pyridine acetate buffer (PyAc) at $\mathrm{pH}=5.0$. A $5 \mathrm{ml}$ aliquot of PyAc was added to the concentrate and the mixture was applied as a layer to the top of the column. PyAc $(50 \mathrm{mM})$ was then eluted through the column $\left(3.4 \mathrm{~atm} \mathrm{~N}_{2}\right)$, splitting the colored PVD extract into three discrete bands. When the first band reached the outlet, the eluent was collected in $10 \mathrm{ml}$ fractions, and the inlet solution was switched to a $750 \mathrm{mM}$ PyAC buffer $(\mathrm{pH}=5.0)$. After the second band had eluted, the inlet solution 
was changed to a $2 \mathrm{mM}$ PyAC buffer $(\mathrm{pH}=5.0)$. After elution of the final band, the solution was flushed with $50 \mathrm{mM}$ PyAC to regenerate the column.

The product purity was evaluated by mapping individual fractions (after appropriate dilution) with fluorescence spectroscopy (excitation 200-450 nm, emission 250-600 nm; Perkin Elmer LS 55 fluorescence photometer). The desired chromophore fluoresces at an emission:excitation peak at 460:405 nm (Parker et al., 2014). Only fractions containing this peak at $>10: 1$ intensity over the background were selected for inclusion in the final product. This fraction proved to be $50 \pm 10 \mathrm{ml}$, collected after discarding an initial $100 \pm 10 \mathrm{ml}$ of eleuent. All of the fractions were eluted with $750 \mathrm{mM}$ PyAc buffer, and amounts to collection of the $50 \mathrm{ml}$ surrounding the middle band, consistent with a previous observations that the front running band contains degradation productions (Meyer and Abdallah, 1978). The selected fractions were pooled and rotary evaporated as described above, resulting in an $80 \%$ volume reduction. The product was then frozen and lyophilized until dry. Based on four replicate trials, solid yield averaged $50 \pm 10 \mathrm{mg} \mathrm{l}^{-1}$ growth medium.

Freeze-dried samples from four replicate trials were dissolved in deionized water to an approximate concentration of $0.25 \mathrm{mg} \mathrm{ml}^{-1}$ and analyzed using Thermo Scientific Dionex UltiMate 3000 LC system equipped with a thermo C18 column $(50 \mathrm{~mm} \times 2.1$ $\mathrm{mm}, 1.9 \mu \mathrm{m}$ particle size) coupled via heated electrospray ionization (HESI) to a mass spectrometer (Thermo Fisher Scientific Exactive Plus MS) operated in positive ion mode at a mass range of 150-2000 amu. Elution of analytes was performed with deionized water $(0.1 \% \quad \mathrm{FAO})$ and acetonitrile $(0.1 \%$ FAO) at a flow rate of $0.5 \mathrm{~mL} / \mathrm{min}$. Chromatograms produced a dominant peak with a $\sim 24$ s retention time. 
Tentative assignments of PVD structure, based on matching masses (Figure 1) to known structures and common motif modifications, are shown in Table 1. Major products included PVD-GB1, an PVD-GB1 -OH $\beta$-elimination product, and sulfonated PVD-GB1, as previously shown for this strain (Parker et al., 2014). In trial A, we also note the possible presence of PVD-D47, which has a different protein backbone. However, masses consistent with an added methyl group and sulfonated versions of both the methyl PVDGB1 (the predominant form in $\mathrm{B}-\mathrm{D}$ ) and the PVD-GB1 -OH $\beta$-elimination product are also present. It should also be noted that each run yielded a different combination of products, and that definite identification of these products requires additional analysis, such as by siderotyping (Fuchs et al., 2001) or tandem mass spectrometry (Budzikiewicz et al., 2007). Although their presence has been recognized in many ringed siderophores (Fuchs et al., 2001; Hickford et al., 2004), the causes for formation and biological significance of sulfonated siderophores are unknown.

The procedure described herein provides a method to produce $\sim 100 \mathrm{mg}$ of purified PVD. It should be noted that the purified PVD obtained here is a complicated mixture of closely related structures. This observation suggests that although the approach was optimized for P. putida GB1, it may be adapted to other Pseudomonads to produce specific PVDs of interest. It may also provide a generalized method for scaling up the procedure for industrial and technological applications (Ahmed and Holmstrom, 2014; Rajkumar et al., 2010).

We thank the National Science Foundation Geobiology and Low-Temperature Geochemistry Program (EAR-0921313) for support. 


\begin{tabular}{cll}
\hline Major Peak $(\mathrm{amu})$ & \multicolumn{1}{c}{ Relative abundance $(\%)$} & Tentative Assignment \\
\hline 1218 & $\mathrm{~A}=100 ; \mathrm{B}=0 ; \mathrm{C}=0 ; \mathrm{D}=16$ & PVD-D47 $^{\mathrm{a}}$ \\
1234 & $\mathrm{~A}=53 ; \mathrm{B}=18 ; \mathrm{C}=35 ; \mathrm{D}=45$ & OH $\beta$-elimination of $1251^{\mathrm{b}}$ \\
1251 & $\mathrm{~A}=15 ; \mathrm{B}=59 ; \mathrm{C}=40 ; \mathrm{D}=30$ & PVD-GB1 $^{\mathrm{b}}$ \\
1265 & $\mathrm{~A}=32 ; \mathrm{B}=13 ; \mathrm{C}=9 ; \mathrm{D}=24$ & methylation of $1251^{2}$ sulfonated 1234 \\
1316 & $\mathrm{~A}=59 ; \mathrm{B}=72 ; \mathrm{C}=32 ; \mathrm{D}=79$ & sulfonated $1251^{\mathrm{b}}$ \\
1332 & $\mathrm{~A}=22 ; \mathrm{B}=100 ; \mathrm{C}=100 ; \mathrm{D}=100$ & sulfonate 1265 \\
1346 & $\mathrm{~A}=26 ; \mathrm{B}=22 ; \mathrm{C}=8 ; \mathrm{D}=16$ & sulfonated 16 \\
\hline
\end{tabular}

Table 1. Tentative assignment of major peak masses of purified siderophores from Figure 1. Relative abundance refers to height of major in Figure 1A-D. Masses with abundance $<20 \%$ in all mass spectra (A-D) are not assigned. ${ }^{\mathrm{a} B u d z i k i e w i c z ~ 2010 ;}{ }^{\mathrm{b}}$ Parker et al. 2014. 


\section{Figures}

Figure 1. Mass spectra of samples from four replicate trials of PVD purification (A-D). Peaks highlighted in gray are tentatively assigned in Table 1. 


\section{References}

Ahmed, E., Holmstrom, S.J.M., 2014. Siderophores in environmental research: roles and applications. Microb Biotechnol 7, 196-208.

Budzikiewicz, H., 2010. Microbial Siderophores, in: Kinghorn, A.D., Falk, H., Kobayashi, J. (Eds.), Fortschritte der Chemie organischer Naturstoffe / Progress in the Chemistry of Organic Natural Products, Vol. 92. Springer Vienna, Vienna, pp. 1-75.

Budzikiewicz, H., Schäfer, M., Fernández, D.U., Matthijs, S., Cornelis, P., 2007. Characterization of the chromophores of pyoverdins and related siderophores by electrospray tandem mass spectrometry. BioMetals 20, 135-144.

Edberg, F., Kalinowski, B.E., Holmstrom, S.J.M., Holm, K., 2010. Mobilization of metals from uranium mine waste: the role of pyoverdines produced by Pseudomonas fluorescens. Geobiology 8, 278-292.

Fuchs, R., Schafer, M., Geoffroy, V., Meyer, J.M., 2001. Siderotyping - A Powerful Tool for the Characterization of Pyoverdines. Current Topics in Medicinal Chemistry 1, 31-57.

Hickford, S.J.H., Küpper, F.C., Zhang, G., Carrano, C.J., Blunt, J.W., Butler, A., 2004. Petrobactin Sulfonate, a New Siderophore Produced by the Marine Bacterium Marinobacter hydrocarbonoclasticus. Journal of Natural Products 67, 1897-1899.

Kraemer, S.M., Duckworth, O.W., Harrington, J.M., Schenkeveld, W.D.C., 2014. Metallophores and Trace Metal Biogeochemistry. Aquat Geochem 21, 159-195.

Kraepiel, A.M., Bellenger, J.P., Wichard, T., Morel, F.M., 2009. Multiple roles of siderophores in free-living nitrogen-fixing bacteria. Biometals 22, 573-581.

Lemanceau, P., Bauer, P., Kraemer, S., Briat, J.F., 2009. Iron dynamics in the rhizosphere as a case study for analyzing interactions between soils, plants and microbes. Plant Soil 321, 513-535.

Meyer, J.-M., 2000. Pyoverdines: pigments, siderophores and potential taxonomic markers of fluorescent Pseudomonas species. Arch. Microbiol. 174, 135-142.

Meyer, J.-M., Stintzi, A., De Vos, D., Cornelis, P., Tappe, R., Taraz, K., Budzikiewicz, H., 1997. Use of Siderophores to Type Pseudomonads: The Three Pseudomonas Aeruginosa Pyoverdine Systems. Microbiology 143, 35-43.

Meyer, J.M., Abdallah, M.A., 1978. The Fluorescent Pigment of Pseudomonas fluorescens: Biosynthesis, Purification and Physicochemical Properties. Microbiology 107, 319-328.

Neilands, J.B., 1995. Siderophores: Structure and Function of Microbial Iron Transport Compounds. J. Biol. Chem. 270, 26723-26726.

Parker, D.L., Lee, S.-W., Geszvain, K., Davis, R.E., Gruffaz, C., Meyer, J.-M., Torpey, J.W., Tebo, B.M., 2014. Pyoverdine synthesis by the $\mathrm{Mn}(\mathrm{II})$-oxidizing bacterium Pseudomonas putida GB-1. Frontiers in Microbiology 5.

Parker, D.L., Morita, T., Mozafarzadeh, M.L., Verity, R., McCarthy, J.K., Tebo, B.M., 2007. Inter-relationships of $\mathrm{MnO}_{2}$ precipitation, siderophore-Mn-(III) complex formation, 
siderophore degradation, and iron limitation in Mn-(II)-oxidizing bacterial cultures. Geochim. Cosmochim. Acta 71, 5672-5683.

Parker, D.L., Sposito, G., Tebo, B.M., 2004. Manganese(III) binding to a pyoverdine siderophore produced by a manganese(II)-oxidizing bacterium. Geochim. Cosmochim. Acta 68, 4809-4820.

Rajkumar, M., Ae, N., Prasad, M.N.V., Freitas, H., 2010. Potential of siderophoreproducing bacteria for improving heavy metal phytoextraction. Trends in Biotechnology 28, 142-149.

Still, W.C., Kahn, M., Mitra, A., 1978. Rapid chromatographic technique for preparative separations with moderate resolution. The Journal of Organic Chemistry 43, 2923-2925. 


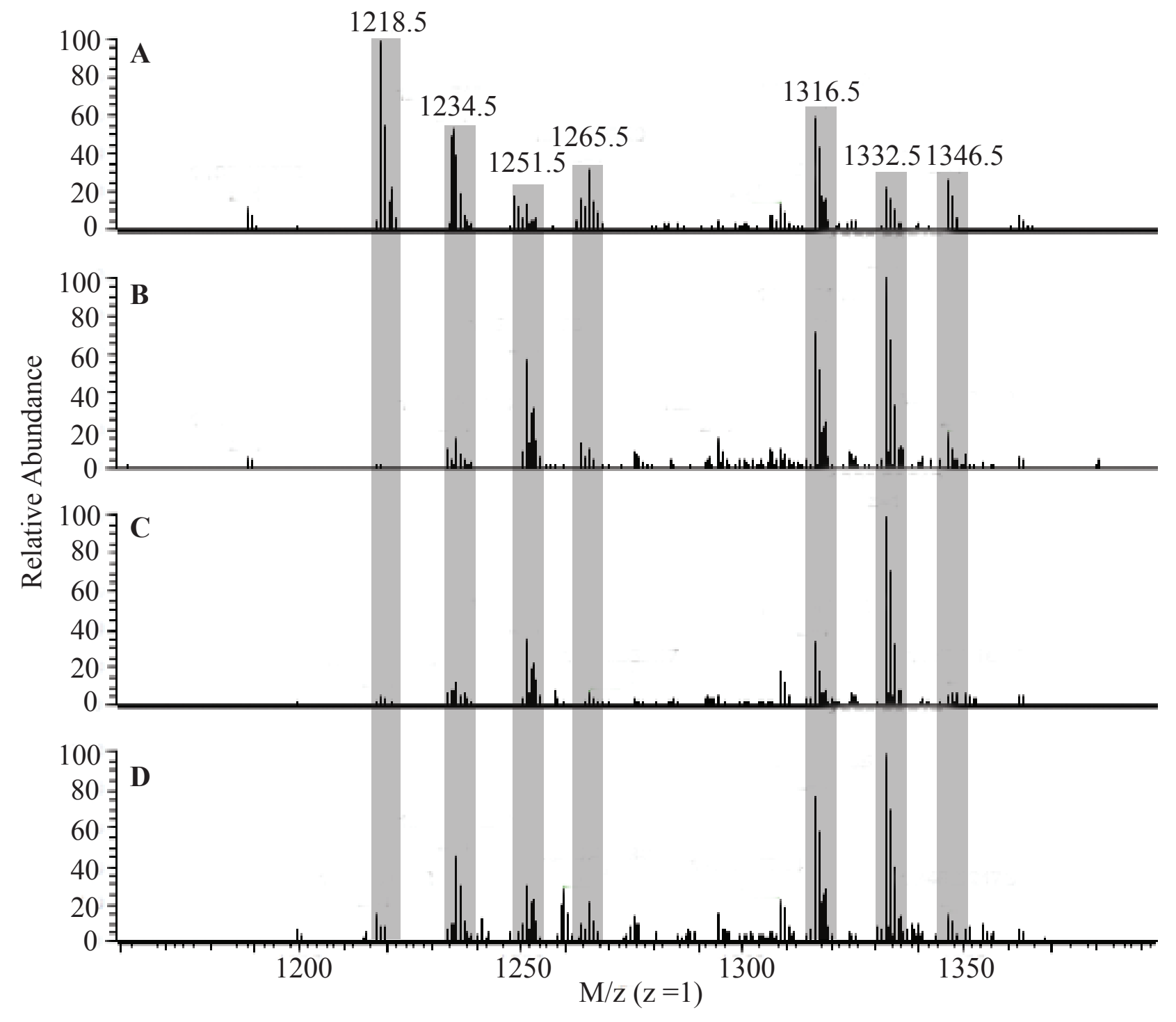

Figure 1. 